\title{
Application of Individualized 3D-Printed Artificial Vertebral Body for Cervicothoracic Reconstruction in a Six-Level Recurrent Chordoma
}

\author{
Xinghai YANG*, Wei WAN*, Haiyi GONG*, Jianru XIAO \\ Second Military Medical University, Changzheng Hospital, Spine Tumor Center, Department of Orthopedic Oncology, Shanghai, China \\ *Xinghai Yang, Wei Wan and Haiyi Gong contributed equally to this work and all should be considered as first authors. \\ Corresponding author: Jianru XIAO jianruxiao83@163.com
}

\section{ABSTRACT}

AIM: To present the surgical planning, manufacturing and implantation of an individualized 3D-printed prosthesis for cervicothoracic reconstruction in a six-level recurrent chordoma.

MATERIAL and METHODS: A 40-year-old female patient with recurrent cervicothoracic chordoma was admitted in our center. Onestage tumorectomy was performed using the combined anterior and posterior approach, and a customized 3D-printed prosthesis was anatomically installed on the defect with vertebral screws from C2 to T2.

RESULTS: The whole procedure took 13 hours, and intraoperative blood loss was $7500 \mathrm{~mL}$. The patient recovered uneventfully, and symptoms of pain and weakness of right upper extremity were significantly diminished postoperatively. No local recurrence was found during 9-month follow-up, and no subsidence, displacement or fracture of the prosthesis was observed radiographically.

CONCLUSION: To the best of our knowledge, this is the first report of biomechanical reconstruction using an individualized 3D-printed artificial vertebral body in a six-level recurrent chordoma. Customized design of the prosthesis helps to address the difficulty in fixation and simplify the surgical procedure. In the spinal transition point where solid fusion is hard to achieve, an individualized 3D-printed implant may exhibit excellent primary stability. Accordingly, this study believes that such burgeoning technique shows a promising prospect in complicated spinal oncology surgery.

KEYWORDS: 3D-Printed, Artificial vertebral body, Cervicothoracic, Six-level, Chordoma

\section{INTRODUCTION}

$\longrightarrow$ hordoma refers to a low-grade malignant tumor from embryonic notochord remnants $(5,18)$. It occurs in the axial skeleton and takes up nearly $4 \%$ of primary bone malignancy $(1,26)$. Clinically, chordoma is characterized by bony involvement, high recurrence rate, local invasion as well as the potential for distant metastases $(34,36)$. The current mainstay treatment for chordoma is en-bloc surgical resection with negative margins $(5,21,29,30)$. Boriani et. al. verified that the prognosis is deeply affected by the appropriateness of the first surgical treatment (5). However, wide or marginal margin excision rate only reaches $21 \%$ in the mobile spine $(1,5)$, and the local recurrence rate remains up to $17-35 \%$ (34).

Chordoma is rarely observed in the cervical spine, taking up only $6 \%$ of all chordomas (3). Clinically, the initial symptom arises when the chordomas have already been progressed to a larger size with paravertebral or spinal canal extension and encapsulation of one or both vertebral arteries (VAs) and nerve roots $(15,29)$. Thus, margin-free en-bloc resection is considered impossible because of these anatomical constraints (14). Multilevel recurrent cervical chordomas raise even more challenges in further surgical treatments, including not only 
the more aggressive feature and incomplete or absent tumor capsule, but also the involvement of dura mater (3). Moreover, extensive defect after multi-level cervical spondylectomy is difficult to reconstruct without using a standard surgical method $(2-4,8,12,24,28)$, and there is always the loss of the cervical physiological curvature.

Individualized implants manufactured for reconstruction of skull defect in human body has been employed for years (7). In contrast, the use of customized 3D-printed prosthesis for spinal surgery has emerged only recently $(16,19,22,23,27,31,33)$. However, it has already exhibited significant advantages in surgical procedures. The superiorities of 3D-printed prosthesis design covers: 1) it fits to the upper and lower vertebrae endplates closely to achieve solid fusion and retain the physiological curvature postoperatively $(23,27) ; 2)$ it is easy to place in the long defect, which facilitates the surgery and simplifies the reconstruction (19); 3) the porous surface could induce bone ingrowth and enhance stability $(16,31,33)$. In this technical note, a unique case of six-level recurrent chordoma from level of C3 to T1 is presented. To the best of our knowledge, it is the first application of individualized 3D-printed artificial vertebral body in revised surgery of cervicothoracic chordoma with the most vertebral levels involvement.

\section{MATERIAL and METHODS}

\section{Case Report}

A 40-year-old female patient was admitted to our center with the weakness of right upper extremity and burning pain in right forearm for 5 months. The symptoms gradually increased, and progressing soft tissue mass appeared on her right neck. History suggested that the patient had received two surgical treatments for cervical spine chordoma previously. MRI and CT scanning confirmed the diagnosis of recurrent chordoma at the level of $\mathrm{C} 3$ to $\mathrm{T} 1$ about 3 years after the previous revision surgery (Figure 1A-D). The vertebral bodies and the posterior elements were osteolytic. The dura was compressed, and the right VA was encapsulated and displaced to the left. The pre-operative CT angiogram of vertebral artery revealed the complete obstruction of the left VA so that the right VA intraoperatively was considered to taken. Pre-operative PET-CT showed no evidence of metastasis. The patient decided to receive surgical treatment as she presented with a rapid and deteriorating neurologic symptoms.

\section{Surgical Planning}

Based on the pre-operative CT angiogram of VA, the patientspecific 3D-Printed model of the cervicothoracic spine can be built with the carotid and vertebral arteries and tumor in place (Figure 2A). It is conducive to gaining insight into the complicated anatomy and providing a reliable reference to yield an adequate surgical margin intraoperatively (32). The tumor was graded as Enneking IB (9), and distributed to layers A-D and sectors 7-4 following the Weinstein-Boriani-Biagini Classification (6). Due to the large volume and wide extent of the recurrent tumor, the total en-bloc spondylectomy was not feasible. Based on the pre-operative imaging studies, intralesional spondylectomy was to be performed in Enneking appropriate manner. Since the six-level vertebras were involved, and no reliable experience of biomechanical reconstruction could be learned from, researchers planned to perform a one-stage intralesional extracapsular excision using anteroposterior approach and to reconstruct the cervico thoracic spine using a customized 3D-printed titanium prosthesis and pedicle screws fixation from level of C1 to T3.

\section{Design and Manufacture of Prosthesis}

According to the individualized anatomical features and expected anterior bone defect, a specific 3D-Printed artificial vertebral body was designed using MIMICS 15.0 (Materialise NV, Leuven, Belgium). The dimensions of the prosthesis were based on preoperative CT. The porous prosthesis was produced from titanium alloy and manufactured using 3D printing technique. The diameters of pores and wires were 800 and $550 \mu \mathrm{m}$, respectively, with an average effective porosity of about $70 \%$. The porosity could induce bone ingrowth as a scaffolding since it is biomechanically similar to normal
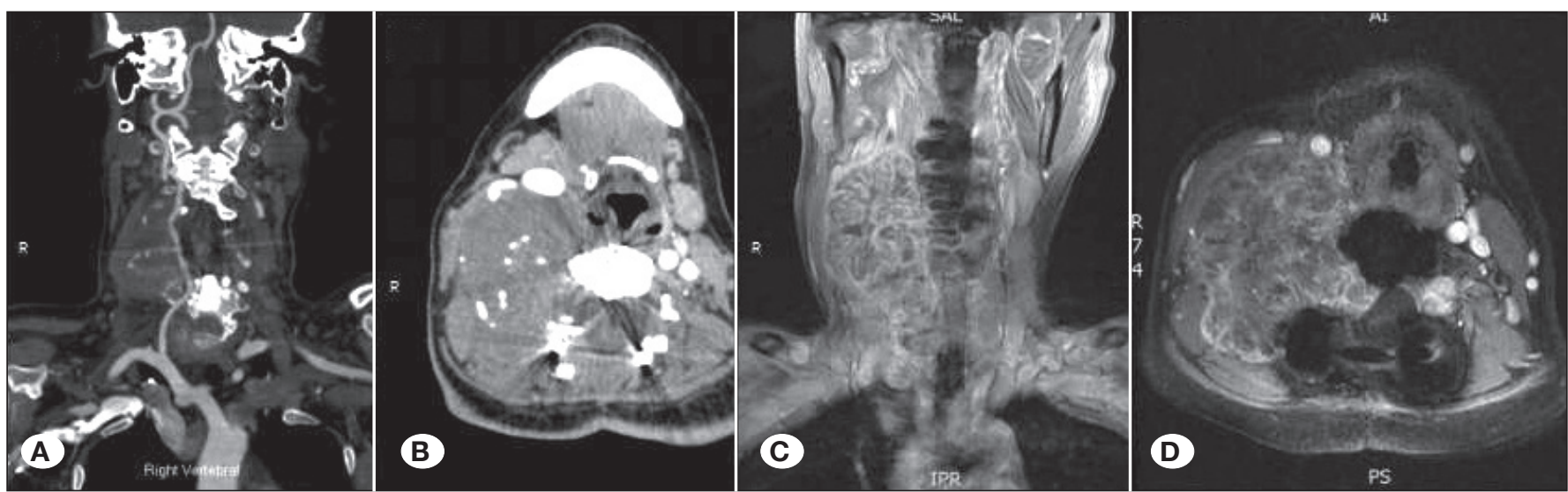

Figure 1: The pre-operative CT angiogram (A: coronal reconstruction, B: axial) of vertebral artery (VA) revealed the extradural soft tissue mass and local osteolytic damage. The dura was compressed and the right vertebral artery was encapsulated and displaced to the left. Furthermore, the left VA had been completely blocked. The tumor with multilevel spine involvement was significantly heterogeneous enhanced on gadolinium-enhanced MRI (C: coronal, D: axial). 
cancellous bone. The titanium alloy powder (Ti-6AL-4V) was completely melted by a selective laser and constituted a tight layer after consolidation. The successive layers were applied roughly $30 \mu \mathrm{m}$ thick as guided by a 3D computer-aided design software. This procedure was repeated until all layers were completed, and the prosthesis was prepared in 3 different sizes to fit the real size of intraoperative bone defect (Figure 2B). The 3D-printed prostheses were fabricated by Naton medical scientific academy. The overall process strictly followed the National Standard of Implants for Surgery in China. Informed consent was gained from the patient and her family with full disclosure on the potential benefits and risks.

\section{Surgical Management}

After general anesthesia, the patient was placed in supine position with the head slightly extended and rotated toward the left side. A combined pre- and retrovascular extraoral approach was employed on right side as previously described (35), and the incision was extended to the suprasternal notch. During the surgery, a paravertebral, grayish $8 \times 7 \times 4 \mathrm{~cm}-$ sized soft mass was found to extend into spinal canal with strong local invasion (Figure $3 \mathrm{~A}$ ). The right vertebral artery and cervical nerve roots were closely encapsulated. The vertebral bodies (C6-T1) and the right posterior elements were destroyed in an osteolytic manner, while the titanium mesh and plate were surrounded by recurrent tumor. because of the proximal vital neurovascular structures, intralesional resection in Enneking appropriate manner was applied. The right VA and nerve roots were rigorously protected and scatheless. The anterior fixation system was removed completely after the soft mass was grossly resected. The osteolytic vertebral bodies (C6, C7 and T1) and the right posterior elements were stepwise excised. The inferior endplate of C2 and the superior endplate of T2 were decorticated, and subsequently the right-size customized 3D-printed artificial vertebral body was implanted to replace the defect. For reconstruction, 3 anterior vertebral screws were inserted into C2 and T2 through reserved holes via a 3-point interlocking pattern, respectively (Figure 3B). Next, the patient was shifted to prone position for the posterior operation. The previous screws and rods were extracted, and we inserted the lateral mass screws of $\mathrm{C} 1$ and pedicle screws of $\mathrm{C} 2, \mathrm{~T} 2$ and $\mathrm{T} 3$. The residual lesion involved posterior elements of $\mathrm{C} 6, \mathrm{C} 7$ and $\mathrm{T} 1$ was completely removed (Figure 3D). Dual diameter rods were employed to offer a solid connection (Figure 3C). The total surgical time was 13 hours, and the intraoperative blood loss was $7500 \mathrm{~mL}$.

\section{Post-operative Care and Recovery}

The patient was transferred to intensive care unit of our department after surgery. She was separated from ventilator 2 days later, and her trachea cannula was removed on the 4th day after surgery. The patient convalesced smoothly without any perioperative complications (e.g. hoarseness, dysphagia and breathing difficulties). Her neurologic function was significantly improved postoperatively, and she could walk 4 weeks later with an assisted cervicothoracic spinal orthosis for 3 months. Histopathological examination and immunohistochemical analysis of the specimen confirmed the diagnosis of chordoma, and the margin was free (Figure 4).

\section{Follow-up}

The follow-up evaluation was performed regularly through clinical visits in the $3^{\text {rd }}, 6^{\text {th }}$ and $9^{\text {th }}$ month after surgery. Though researchers had strongly recommended proton-beam and
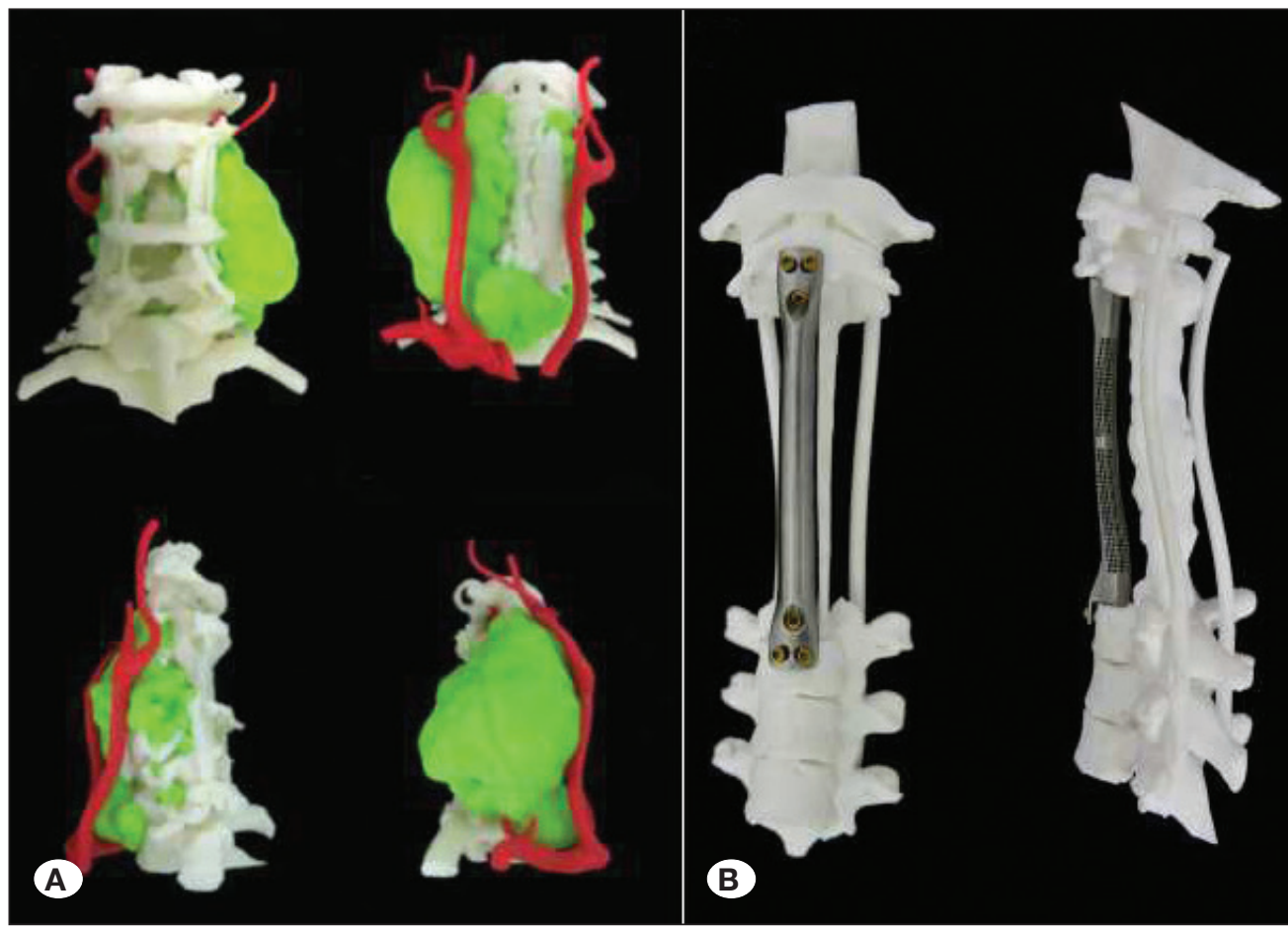

Figure 2: A) Preoperative 3D-printed model shows the tumor involving the vertebral bodies and bilateral pedicles, lateral masses and transverse processes from C3 to $\mathrm{T} 1$ with strong local invasion. The 3D-printed model also reveals the proximity to vital neurovascular structures. B) The individualized 3D-Printed artificial vertebral body with a specific porous titanium structure. The front surface is smooth to prevent adhesion with aorta and esophagus. The anterior pre-angled screw holes were designed in a 3-point interlocking pattern to achieve greater pullout strength and better stability. 


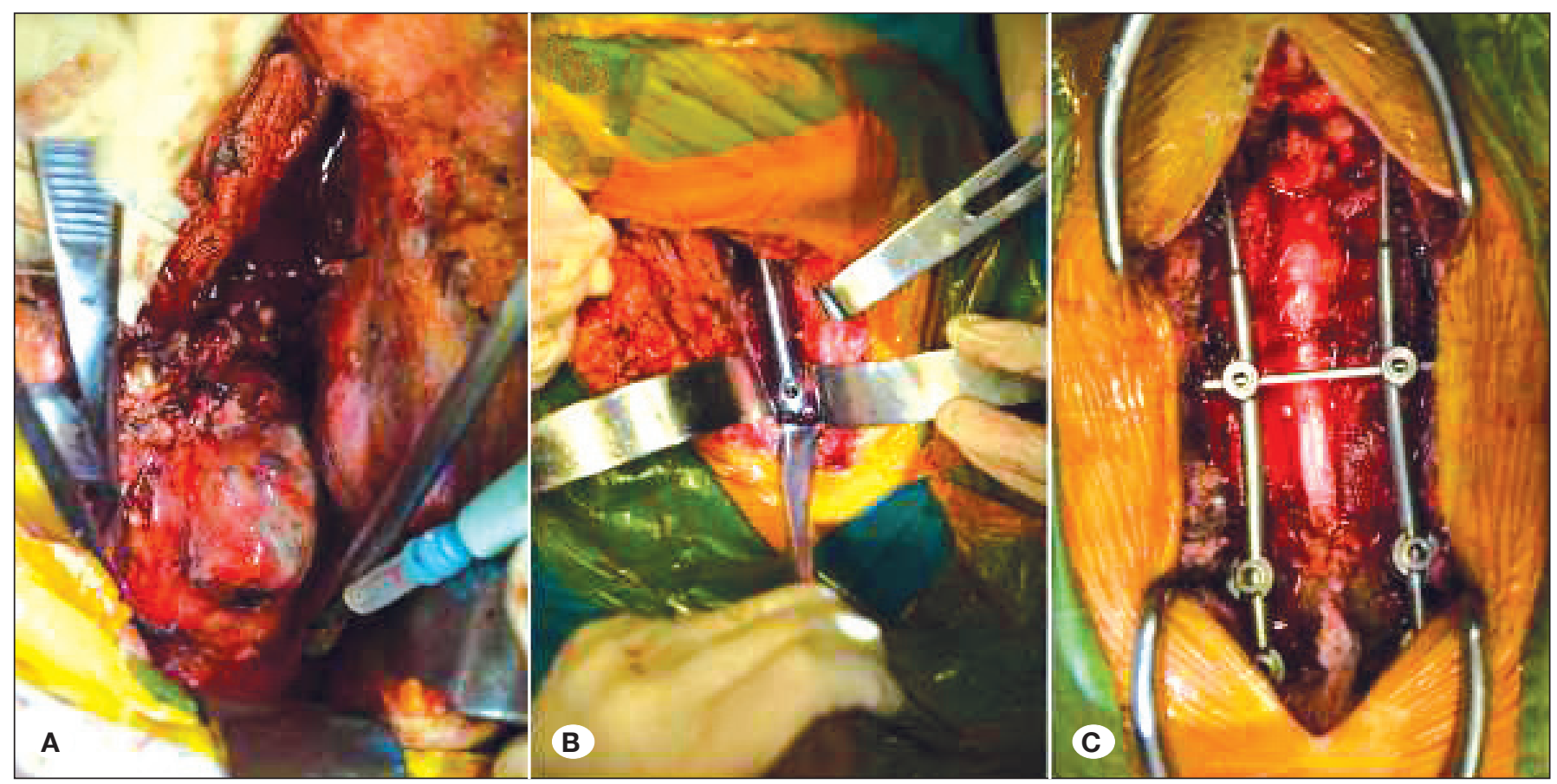

Figure 3: A) The appearance of the neoplasm intraoperatively. Intralesional extracapsular excision in Enneking appropriate manner was employed to resect the tumor and rigid reconstruction was achieved with the individualized 3D-Printed prosthesis (B), and posterior screw rod system (C).

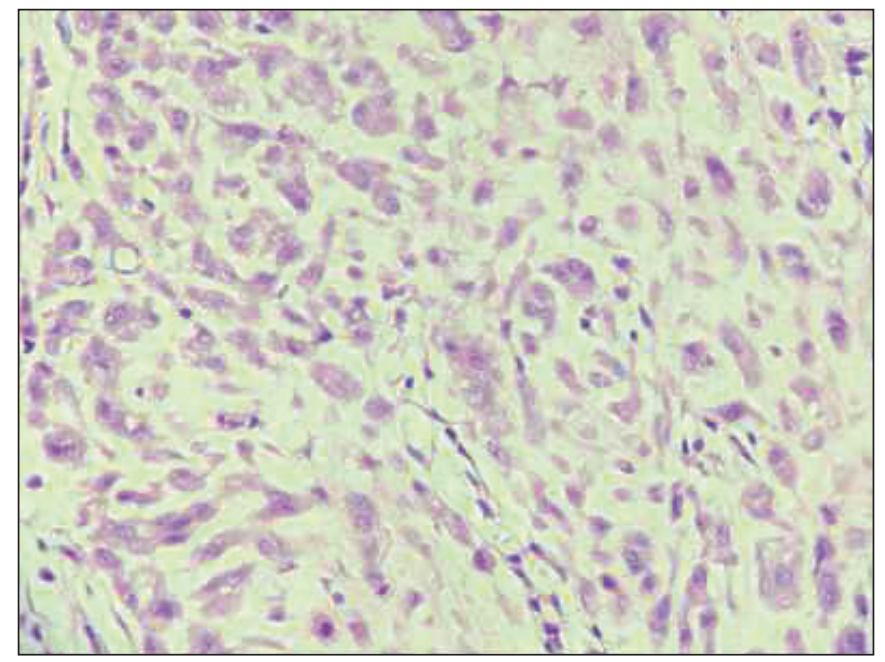

Figure 4: Histopathological examination of the specimen confirmed the diagnosis of chordoma (Hematoxylin and eosin $\times 400)$.

heavy ions radiation therapy as complementary treatment, the patient had to give up the attempt for financial reason. Plain radiographs performed at 3 months after surgery showed a stable C1 to T3 instrumentation. No local recurrence was detected by contrast-enhanced CT at the $3^{\text {rd }}$ and $9^{\text {th }}$ month respectively. Moreover, no subsidence or dislocation or fractures of the 3D-printed artificial vertebral body and screwrod system was observed. Compared with the CT scanning performed in the $3^{\text {rd }}$ month, the latest scanning clearly showed the bone of posterior C2 and T2 vertebral bodies ingrowth towards the porous structure of the prosthesis (Figure 5A-F).

\section{DISCUSSION}

Chordoma is considered the most common primary spine malignancy, and it is generally insensitive to adjuvant treatment such as chemotherapy and conventional radiation therapy $(13,20,34,36)$. Besides, inadequate intralesional resection inevitably results in higher local recurrence rates (1). Surgical resection in margin-free en-bloc fashion is considered the most ideal treatment for better local control and clinical outcomes of patients $(5,18,26)$. However, the anatomical constraints and high demanding surgical skill and techniques make it relatively challenging in the treatment of cervical chordoma $(14,15)$.

Because of its rarity, only few reports about the surgical treatment of cervical chordoma involving equal to or greater than 3 levels have been published $(2-4,8,12,24,28)$. Excision of multilevel cervical chordoma in Enneking appropriate manner (11), and anterior reconstruction for long defect present extreme technical demands, especially for those with upper cervical spine involvement $(18,30)$. Appropriate biomechanical reconstructions always cause fewer complications and reduce the possibility of revision surgery (10). However, the unique anatomical and morphological characteristics limit a uniform standard method to reconstruct. Conventional anterior reconstructive methods for multilevel cervical chordoma could fall into five types, namely autogenous bone graft (2), autogenous bone graft combined with titanium plate (24), titanium mesh cage (28), titanium mesh cage and plate system (8), fabricated cage system (12). The main drawbacks 

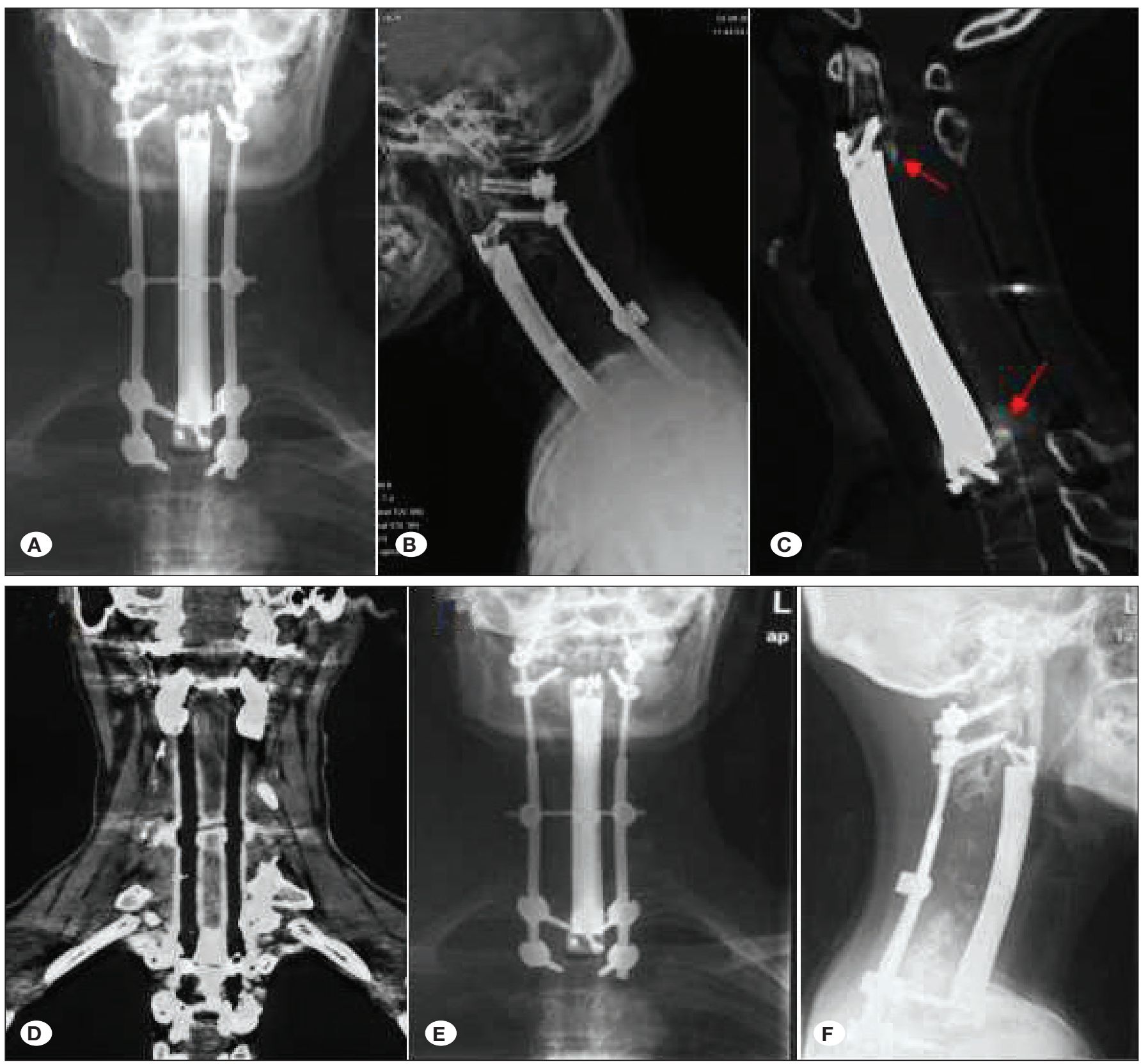

Figure 5: Postoperative radiographs (A: anteroposterior, B: lateral) at the 3-month follow-up point after surgery show the instrumentation from C1 to T3 was stable. No subsidence or dislocation or fractures of the 3D-printed implant, screws or rods was observed. No evidence of local recurrence was detected through contrast-enhanced CT (C: sagittal reconstruction, D: coronal reconstruction) at the $9^{\text {th }}$ month. The CT scanning clearly revealed the bone of posterior C2 and T2 vertebral bodies ingrowth towards the porous structure of the prosthesis ( $\mathrm{C}$, arrowheads). The latest $\mathrm{X}$-ray (E: anteroposterior, F: lateral) performed at 13 months indicated the internal fixations were stable.

of conventional methods are poor individual matching and limited biomechanical strength. Therefore, it is common to see straightened spinal physiological curvature or subsidence or dislocation of implant at mid to long-term follow-up. Moreover, conventional anterior reconstructive techniques usually require sufficient bone grafts. However, autogenous bone graft generally brings about persistent pain and motor function limitation, while allogenous bone or artificial bone graft leads to increased medical costs (33).
In our case, the recurrent cervicothoracic chordoma involved six-level which makes it difficult to reconstruct such a large defect. The length of existing regular titanium mesh cage and plate does not meet the surgical requirements. Furthermore, at the cervicothoracic junction exists an anatomically hostile area in which there exists a mobile to non-mobile transition point and an anatomical lordosis to kyphosis transition (10). Thus, the solid fusion is usually hard to achieve using conventional reconstructive approaches. As a result, it is vital 
to find better ways to reconstruct the defect after a multilevel vertebrectomy.

The 3D printing process was first proposed by Charles Hull in 1986 (19). It basically refers to creating 3D object from a digital design by successive layering material under computer control. Current applications of 3D-Printing in spine surgery consists of the following aspects: surgical planning and pre-training, surgical instrumentation, implantable devices, and tissue engineering $(16,19,22,23,27,31,33)$. However, the use of individualized 3D-printed implant in spinal oncology has only emerged in recent years with the results proven promising $(16,19,31,33)$. The customized prosthesis can fit the complex shapes precisely, minimize dead space (16) and facilitate osseointegration without additional grafting $(16,19,22,23,27,31,33)$. It helps to simplify the surgical technique and reduce the operating time.

The superiorities and specificities of our 3D-printed prosthesis design covered: (1) The posterior porous surface could trigger osseointegration and enhance stability; (2) the prosthesis retains the physiological curvature postoperatively with zeroprofile to avoid dysphagia; (3) be different from traditional titanium mesh, the better biomechanical matching and load-bearing facilitated the 3D-printed implant to resist the subsidence or dislocation; (4) the anterior vertebral screws $(\mathrm{C} 2, \mathrm{~T} 2)$ were implanted in a 3-point interlocking pattern to achieve greater pullout strength and better rotational stability than titanium mesh cage system.

Though 3D-printed prosthesis has many advantages and great prospect in spinal oncology, there are still many limitations to restrict its application at present. First, the manufacturing process of individualized 3D-Printed prosthesis is very complicated owing to the elaborate and complex anatomy of spine (27). The computer-aided design software is usually sophisticated, and the machinery is not easily accessible (22). Besides that, implant modelling always requires specific skills which are lacked in most surgical departments (17). Second, the commercial costs of 3D-printed prosthesis are high $(19,27)$, and the long manufacturing time still restrict the expanded implementation of the technology in hospitals (23). Third, though the implant is generally prepared in different sizes, intraoperative modification is still confined because of the limited extension capabilities (16). Fourth, the strength of 3D-printed implants may not be guaranteed (16), while the long-term follow-up in comparison to conventional techniques is also absent. Finally, there is an urgent need for the uniform regulatory framework to standardize the registration, production and implantation of the prosthesis (22).

In brief, the application of individualized 3D-printed implants in spinal oncology is clearly in the ascendant. Accordingly, there are many aspects that should be improved and perfected. Further research is needed to enhance the time- and costeffectiveness of this promising technology for medical intervention (23). Finite element analysis should be conducted to improve the porous metal structure continuously for preferable dynamic load-bearing (23). Moreover, the burning questions also include: the combination of 3D-printed implants and biologics, the development of bioceramic composites as well as the use of bioresorbable materials $(19,23)$. Research on these hotspot issues is ongoing and we believe that international standardization process would speed up the development of individualized 3D-Printing technology.

\section{CONCLUSION}

To the best of our knowledge, this is the first report of biomechanical reconstruction using an individualized 3Dprinted artificial vertebral body in the surgical treatment of a six-level recurrent chordoma. The individualized 3D-printed prosthesis was implanted to replace the defect in high accuracy without additional bone grafting. It was better loadbearing to resist the subsidence or dislocation, which can reduce the rate of revision surgeries effectively in the longterm. Though several current limitations have limited the wide application of this technology, customized 3D-printed prosthesis implantation still shows a promising prospect in complicated spinal oncology surgery.

\section{REFERENCES}

1. Ailon T, Torabi R, Fisher CG, Rhines LD, Clarke MJ, Bettegowda C, Boriani S, Yamada YJ, Kawahara N, Varga PP, Shin JH, Saghal A, Gokaslan ZL: Management of locally recurrent chordoma of the mobile spine and sacrum: A systematic review. Spine 41 Suppl 20: S193-S198, 2016

2. Bailey CS, Fisher CG, Boyd MC, Dvorak MF: En bloc marginal excision of a multilevel cervical chordoma. Case report. J Neurosurg Spine 4(5):409-414, 2006

3. Barrenechea IJ, Perin NI, Triana A, Lesser J, Costantino P, Sen C: Surgical management of chordomas of the cervical spine. J Neurosurg Spine 6(5):398-406, 2007

4. Barrey C, Saint-Pierre G, Frappaz D, Hermier M, Mottolese C: Complete removal of an intraspinal and extraspinal cervical chordoma in one stage using the lateral approach. Technical note. J Neurosurg Spine 5(5):471-475, 2006

5. Boriani S, Bandiera S, Biagini R, Bacchini P, Boriani L, Cappuccio M, Chevalley F, Gasbarrini A, Picci P, Weinstein $\mathrm{JN}$ : Chordoma of the mobile spine: Fifty years of experience. Spine 31(4):493-503, 2006

6. Boriani S, Weinstein JN, Biagini R: Primary bone tumors of the spine. Terminology and surgical staging. Spine 22(9):10361044, 1997

7. Cabraja M, Klein M, Lehmann TN (2009) Long-term results following titanium cranioplasty of large skull defects. Neurosurg Focus 26(6): E10

8. Chou D, Acosta F Jr, Cloyd JM, Ames CP: Parasagittal osteotomy for en bloc resection of multilevel cervical chordomas. J Neurosurg Spine 10(5):397-403, 2009

9. Enneking WF, Spanier SS, Goodman MA: A system for the surgical staging of musculoskeletal sarcoma. Clin Orthop Relat Res 153:106-120, 1980

10. Glennie RA, Rampersaud YR, Boriani S, Reynolds JJ, Williams R, Gokaslan ZL, Schmidt MH, Varga PP, Fisher CG: A systematic review with consensus expert opinion of best reconstructive techniques after osseous en bloc spinal column tumor resection. Spine 41 Suppl 20: S205-S211, 2016 
11. Gokaslan ZL, Zadnik PL, Sciubba DM, Germscheid N, Goodwin CR, Wolinsky JP, Bettegowda C, Groves ML, Luzzati A, Rhines LD, Fisher CG, Varga PP, Dekutoski MB, Clarke MJ, Fehlings MG, Quraishi NA, Chou D, Reynolds JJ, Williams RP, Kawahara N, Boriani S: Mobile spine chordoma: Results of 166 patients from the AOSpine Knowledge Forum Tumor database. J Neurosurg Spine 24(4):644-651, 2016

12. Guppy KH, Chakrabarti I, Isaacs RS, Jun JH: En bloc resection of a multilevel high-cervical chordoma involving C-2: New operative modalities. J Neurosurg Spine 19(2):232-242, 2013

13. Holliday EB, Mitra HS, Somerson JS, Rhines LD, Mahajan A, Brown PD, Grosshans DR: Postoperative proton therapy for chordomas and chondrosarcomas of the spine. Spine 40(8): 544-549, 2015

14. Hsieh PC, Gallia GL, Sciubba DM, Bydon A, Marco RA, Rhines L, Wolinsky JP, Gokaslan ZL: En bloc excisions of chordomas in the cervical spine: review of five consecutive cases with more than 4-year follow-up. Spine 36(24): E1581-1587, 2011

15. Jiang L, Liu ZJ, Liu XG, Ma QJ, Wei F, Lv Y, Dang GT: Upper cervical spine chordoma of C2-C3. Eur Spine J 18(3):293-298, discussion 298-300, 2009

16. Kim D, Lim JY, Shim KW, Han JW, Yi S, Yoon DH, Kim KN, Ha Y, Ji GY, Shin DA: Sacral reconstruction with a 3D-printed implant after hemisacrectomy in a patient with sacral osteosarcoma: 1-year follow-up result. Yonsei Med J 58(2): 453-457, 2017

17. Martelli N, Serrano C, van den Brink H, Pineau J, Prognon P, Borget I, El Batti S: Advantages and disadvantages of 3-dimensional printing in surgery: A systematic review. Surgery 159:1485-500, 2016

18. Meng T, Yin H, Li B, Li Z, Xu W, Zhou W, Cheng M, Wang J, Zhou L, Yang X, Liu T, Yan W, Song D, Xiao J: Clinical features and prognostic factors of patients with chordoma in the spine: A retrospective analysis of 153 patients in a single center. Neuro Oncol 17(5):725-732, 2015

19. Mobbs RJ, Coughlan M, Thompson R, Sutterlin CE 3rd, Phan $\mathrm{K}$ : The utility of $3 \mathrm{D}$ printing for surgical planning and patientspecific implant design for complex spinal pathologies: Case report. J Neurosurg Spine 6(4):513-518, 2017

20. Molina CA, Ames CP, Chou D, Rhines LD, Hsieh PC, Zadnik PL, Wolinsky JP, Gokaslan ZL, Sciubba DM: Outcomes following attempted en bloc resection of cervical chordomas in the C-1 and $\mathrm{C}-2$ region versus the subaxial region: $\mathrm{A}$ multiinstitutional experience. J Neurosurg Spine 21(3):348-356, 2014

21. Pennicooke B, Laufer I, Sahgal A, Varga PP, Gokaslan ZL, Bilsky $\mathrm{MH}$, Yamada $\mathrm{YJ}$ : Safety and local control of radiation therapy for chordoma of the spine and sacrum: A systematic review. Spine 41 Suppl 20: S186-S192, 2016

22. Phan K, Sgro A, Maharaj MM, D'Urso P, Mobbs RJ: Application of a 3D custom printed patient specific spinal implant for $\mathrm{C} 1 / 2$ arthrodesis. J Spine Surg 2(4):314-318, 2016

23. Provaggi E, Leong JJ, Kalaskar DM: Applications of 3D printing in the management of severe spinal conditions. Proc Inst Mech Eng H 231(6):471-486, 2017
24. Rhines LD, Fourney DR, Siadati A, Suk I, Gokaslan ZL: En bloc resection of multilevel cervical chordoma with C-2 involvement. Case report and description of operative technique. J Neurosurg Spine 2(2):199-205, 2005

25. Rotaru H, Stan H, Florian IS, Schumacher R, Park YT, Kim SG, Chezan H, Balc N, Baciut M: Cranioplasty with custom-made implants: Analyzing the cases of 10 patients. J Oral Maxillofac Surg 70(2):e169-e176, 2012

26. Sciubba DM, Chi JH, Rhines LD, Gokaslan ZL: Chordoma of the spinal column. Neurosurg Clin N Am 19:5-15, 2008

27. Spetzger U, Frasca M, König SA: Surgical planning, manufacturing and implantation of an individualized cervical fusion titanium cage using patient-specific data. Eur Spine $\mathrm{J}$ 25(7):2239-2246, 2016

28. Suchomel P, Buchvald P, Barsa P, Froehlich R, Choutka O, Krejzar Z, Sourkova P, Endrych L, Dzan L: Single-stage total C-2 intralesional spondylectomy for chordoma with threecolumn reconstruction. Technical note. J Neurosurg Spine 6(6):611-618, 2007

29. Wang Y, Xiao J, Wu Z, Huang Q, Huang W, Zhu Q, Lin Z, Wang $\mathrm{L}$ : Primary chordomas of the cervical spine: A consecutive series of 14 surgically managed cases. J Neurosurg Spine 17(4):292-299, 2012

30. Wang Y, Xu W, Yang X, Jiao J, Zhang D, Han S, Xiao J: Recurrent upper cervical chordomas after radiotherapy: Surgical outcomes and surgical approach selection based on complications. Spine 38(18):E1141-1148, 2012

31. Wei R, Guo W, Ji T, Zhang Y, Liang H: One-step reconstruction with a 3D-printed, custom-made prosthesis after total en bloc sacrectomy: A technical note. Eur Spine J 26(7):1902-1909, 2017

32. Xiao JR, Huang WD, Yang XH, Yan WJ, Song DW, Wei HF, Liu TL, Wu ZP, Yang C: En bloc resection of primary malignant bone tumor in the cervical spine based on 3-dimensional printing technology. Orthop Surg 8(2):171-178, 2016

33. Xu N, Wei F, Liu X, Jiang L, Cai H, Li Z, Yu M, Wu F, Liu $Z$ : Reconstruction of the upper cervical spine using a personalized 3d-printed vertebral body in an adolescent with ewing sarcoma. Spine 41(1): E50-54, 2016

34. Yang J, Yang X, Miao W, Jia Q, Wan W, Meng T, Wu Z, Cai $X$, Song D, Xiao J: Spine extra-osseous chordoma mimicking neurogenic tumors: Report of three cases and review of the literatures. World J Surg Oncol 14(1):206, 2016

35. Yang X, Huang W, Xiao J, Wu Z, Feng D, Zheng W, Chen H, Liu T, Xie N, Yuan W: Combined pre- and retrovascular extraoral approach for tumors at lateral mass of the atlas. Spine 36(2): 129-136, 2011

36. Zhong N, Yang X, Yang J, Meng T, Yang C, Yan W, Xiao J: Surgical consideration for adolescents and young adults with cervical chordoma. Spine 42(10): E609-E616, 2017 